Article

\title{
Nontrivial Solutions for a System of Fractional $q$-Difference Equations Involving $q$-Integral Boundary Conditions
}

\author{
Yaohong Li ${ }^{1}$, Jie Liu ${ }^{2, *}$, Donal $\mathrm{O}^{\prime} \operatorname{Regan}^{3}$ and Jiafa $\mathrm{Xu}^{4}$ \\ 1 School of Mathematics and Statistics, Suzhou University, Suzhou 234000, Anhui, China; \\ 101023@ahszu.edu.cn \\ 2 School of Mathematics and Information Science, Henan Polytechnic University, \\ Jiaozuo 454000, Henan, China \\ 3 School of Mathematics, Statistics and Applied Mathematics, National University of Ireland, \\ H91 CF50 Galway, Ireland; donal.oregan@nuigalway.ie \\ 4 School of Mathematical Sciences, Chongqing Normal University, Chongqing 401331, China; \\ 20150028@cqnu.edu.cn \\ * Correspondence: jzyzliujie@hpu.edu.cn
}

Received: 24 April 2020; Accepted: 13 May 2020; Published: 20 May 2020

\begin{abstract}
In this paper, we study the existence of nontrivial solutions for a system of fractional $q$-difference equations involving $q$-integral boundary conditions, and we use the topological degree to establish our main results by considering the first eigenvalue of some associated linear integral operators.
\end{abstract}

Keywords: fractional $q$-difference equations; $q$-integral boundary conditions; topological degree; nontrivial solutions

\section{Introduction}

The initial work of $q$-difference calculus can be dated back to Jackson [1,2] and for results on fractional $q$-difference calculus or quantum calculus we refer the reader to [3-28] and the references therein. For example, in $[3,4]$ the author studied some basic properties of fractional $q$-fractional integral and differential operators, and used fixed point theorems in cones to investigate the existence of nontrivial solutions for the fractional $q$-difference equation

$$
\left(D_{q}^{\alpha} y\right)(x)=-f(x, y(x)), 0<x<1,
$$

with the boundary conditions

$$
y(0)=y(1)=0, \text { as } \alpha \in(1,2],
$$

or

$$
y(0)=\left(D_{q} y\right)(0)=0,\left(D_{q} y\right)(1)=\beta \geq 0, \text { as } \alpha \in(2,3] .
$$

In [5] the author considered the three-point boundary value problem of fractional $q$-difference equations

$$
\left\{\begin{array}{l}
D_{q}^{\alpha}+f(t, x(t), x(t))+g(t, x(t))=0,0<t<1, \\
x(0)=D_{q} x(0)=0, D_{q} x(1)=\beta D_{q} x(\eta),
\end{array}\right.
$$

where $\beta \eta^{\alpha-2} \in(0,1), q \in(0,1), \alpha \in(2,3)$ and based on fixed point theorems on mixed monotone operators, some sufficient conditions are used to guarantee the existence and uniqueness of positive 
solutions for the above problem. In [6] the authors discussed the following nonhomogeneous boundary value problem with fractional $q$-derivatives

$$
\left\{\begin{array}{l}
\left(D_{q}^{\alpha} u\right)(t)+f(t, u(t))=0, t \in(0,1), \\
u(0)=\left(D_{q} u\right)(0)=0, \gamma\left(D_{q} u\right)(1)+\beta\left(D_{q}^{2} u\right)(1)=\lambda,
\end{array}\right.
$$

where $q \in(0,1), 2<\alpha \leq 3, \gamma \geq 0, \beta>0$, and $\lambda$ is a parameter. Using the generalized Banach contraction principle and Krasnoselskii's fixed point theorem, uniqueness, existence, and multiplicity of positive solutions for the above problem were obtained in terms of explicit intervals for the nonhomogeneous term.

Coupled systems of fractional $q$-difference equations were investigated in [23-28] (also see [29-35]). In [23] the authors studied the following system of fractional $q$-difference equations with four-point boundary conditions

$$
\left\{\begin{array}{l}
D_{q}^{\alpha} u(t)+f(t, v(t))=0,0<t<1, \\
D_{q}^{\beta} v(t)+g(t, u(t))=0,0<t<1, \\
u(0)=0, u(1)=\gamma_{1} u\left(\eta_{1}\right) \\
v(0)=0, v(1)=\gamma_{2} u\left(\eta_{2}\right)
\end{array}\right.
$$

where $0<q<1,1<\beta \leq \alpha \leq 2,0<\eta_{1}, \eta_{2}<1,0<\gamma_{1} \eta_{1}^{\alpha-1}<1,0<\gamma_{2} \eta_{2}^{\beta-1}<1$ and using the monotone iterative approach they constructed two convergent monotone iterative schemes and obtained two positive solutions for the above problem. In [24] the authors studied the coupled system of fractional $q$-integro-difference equations with nonlocal fractional $q$-integral boundary conditions

$$
\left\{\begin{array}{l}
D_{q}^{\alpha} x(t)=f\left(t, x(t), I_{r}^{\delta} y(t)\right), t \in[0, T], 1<\alpha \leq 2 \\
D_{p}^{\beta} y(t)=g\left(t, y(t), I_{z}^{\varepsilon} x(t)\right), t \in[0, T], 1<\beta \leq 2 \\
x(0)=0, \lambda_{1} I_{m}^{\gamma} x(\eta)=I_{n}^{\kappa} y(\xi) \\
y(0)=0, \lambda_{2} I_{h}^{u} y(\theta)=I_{k}^{v} x(\tau)
\end{array}\right.
$$

where $0<p, q, r, z, m, n, h, k<1, \eta, \xi, \theta, \tau \in(0, T), \delta, \varepsilon, \gamma, \kappa, \mu, v>0$, and $\lambda_{1}, \lambda_{2} \in \mathbb{R}$ are given constants, $I_{\phi}^{\psi}$ is the fractional $\phi$-integral of order $\psi$ with $\phi \in\{r, z, m, n, h, k\}$ and $\psi \in\{\delta, \varepsilon, \gamma, \kappa, \mu, v\}$. Using the Banach contraction principle and the Leray-Schauder alternative, they obtained existence and uniqueness of solutions under some appropriate conditions on $f, g$.

Motivated by the mentioned works above, in this paper we use topological degree theory to study nontrivial solutions for the following system of fractional $q$-difference equations with $q$-integral boundary conditions:

$$
\left\{\begin{array}{l}
D_{q}^{\alpha} x(t)+f_{1}(t, y(t))=0, t \in(0,1) \\
D_{q}^{\alpha} y(t)+f_{2}(t, x(t))=0, t \in(0,1) \\
x(0)=0, D_{q} x(0)=0, D_{q}^{v} x(1)=\int_{0}^{1} h(t) D_{q}^{v} x(t) d_{q} t \\
y(0)=0, D_{q} y(0)=0, D_{q}^{v} y(1)=\int_{0}^{1} h(t) D_{q}^{v} y(t) d_{q} t
\end{array}\right.
$$

where $\alpha \in(2,3), v \in(1,2), D_{q}^{\alpha}$ is the $\alpha$-order Riemann-Liouville's fractional $q$-derivative.

Now, we list our assumptions for $h, f_{i}(i=1,2)$ :

Hypothesis $1(\mathrm{H} 1) . h \geq 0$ and $1-\int_{0}^{1} h(t) t^{\alpha-v-1} d_{q} t:=A>0$. 
Hypothesis 2 (H2). $f_{i} \in C([0,1] \times \mathbb{R}, \mathbb{R})$.

Hypothesis $3(\mathrm{H} 3)$. There exist $b_{i}(t), c_{i}(t) \in C\left([0,1], \mathbb{R}^{+}\right)$with $c_{i}(t) \not \equiv 0$ and $K_{1}(y), K_{2}(x) \in C\left[\mathbb{R}, \mathbb{R}^{+}\right]$ such that

$$
f_{1}(t, y) \geq-b_{1}(t)-c_{1}(t) K_{1}(y), f_{2}(t, x) \geq-b_{2}(t)-c_{2}(t) K_{2}(x), \forall x, y \in \mathbb{R}, t \in[0,1], i=1,2 .
$$

Hypothesis 4 (H4). $\lim _{|y| \rightarrow+\infty} \frac{K_{1}(y)}{|y|}=0, \lim _{|x| \rightarrow+\infty} \frac{K_{2}(x)}{|x|}=0$.

Hypothesis 5 (H5). $\lim \inf _{|y| \rightarrow+\infty} \frac{f_{1}(t, y)}{|y|}>\lambda_{1}, \liminf _{|x| \rightarrow+\infty} \frac{f_{2}(t, x)}{|x|}>\lambda_{1}$, uniformly for $t \in[0,1]$.

Hypothesis 6 (H6). $\lim \sup _{|y| \rightarrow 0} \frac{\left|f_{1}(t, y)\right|}{|y|}<\lambda_{1}, \lim \sup _{|x| \rightarrow 0} \frac{\left|f_{2}(t, x)\right|}{|x|}<\lambda_{1}$, uniformly for $t \in[0,1]$, where $\lambda_{1}$ is the first eigenvalue of the following eigenvalue problem

$$
\left\{\begin{array}{l}
D_{q}^{\alpha} x(t)+\lambda x(t)=0, t \in(0,1) \\
x(0)=0, D_{q} x(0)=0, D_{q}^{v} x(1)=\int_{0}^{1} h(t) D_{q}^{v} x(t) d_{q} t
\end{array}\right.
$$

where $\lambda$ is a parameter, and $\alpha, v, h$ are as in (8).

Finally, we state our main result in this paper:

Theorem 1. Suppose that (H1)-(H6) hold. Then (8) has at least one nontrivial solution.

Remark 1. In (H1), the function $h$ is a non-negative function on $[0,1]$ (it also can be a zero function). If $h \neq 0$ on $[0,1]$, we demand that $\int_{0}^{1} h(t) t^{\alpha-v-1} d_{q} t \in[0,1)$.

\section{Preliminaries}

Let $q \in(0,1)$ and define

$$
[a]_{q}=\frac{1-q^{a}}{1-q}, \quad a \in \mathbb{R}
$$

The $q$-analogue of the power function $(a-b)^{n}$ with $n \in \mathbb{N}_{0}$ is

$$
(a-b)^{0}=1, \quad(a-b)^{n}=\prod_{k=0}^{n-1}\left(a-b q^{k}\right), \quad n \in \mathbb{N}, \quad a, b \in \mathbb{R} .
$$

More generally, if $\alpha \in \mathbb{R}$, then

$$
(a-b)^{(\alpha)}=a^{\alpha} \prod_{n=0}^{\infty} \frac{a-b q^{n}}{a-b q^{\alpha+n}} .
$$

Please note that if $b=0$ then $a^{(\alpha)}=a^{\alpha}$. The $q$-gamma function is defined by

$$
\Gamma_{q}(x)=\frac{(1-q)^{(x-1)}}{(1-q)^{x-1}}, \quad x \in \mathbb{R} \backslash\{0,-1,-2, \ldots\},
$$

and satisfies $\Gamma_{q}(x+1)=[x]_{q} \Gamma_{q}(x)$. 
Definition 1 (see [3], Definition 2.2). Let $\alpha \geq 0$ and $f$ be a function defined on [0,1]. The fractional $q$-integral of the Riemann-Liouville type is $\left(I_{q}^{0} f\right)(x)=f(x)$ and

$$
\left(I_{q}^{\alpha} f\right)(x)=\frac{1}{\Gamma_{q}(\alpha)} \int_{0}^{x}(x-q t)^{(\alpha-1)} f(t) d_{q} t, \quad \alpha>0, \quad x \in[0,1] .
$$

The fractional q-derivative of order $\alpha \geq 0$ is defined by $\left(D_{q}^{0} f\right)(x)=f(x)$ and $\left(D_{q}^{\alpha} f\right)(x)=$ $\left(D_{q}^{m} I_{q}^{m-\alpha} f\right)(x)$ for $\alpha>0$, where $m$ is the smallest integer greater or equal than $\alpha$.

Lemma 1 (see [3], Lemma 2.3). Let $\alpha, \beta \geq 0$ and $f$ be a function defined on $[0,1]$. Then, the next formulas hold:

(i) $\left(I_{q}^{\beta} I_{q}^{\alpha} f\right)(x)=\left(I_{q}^{\alpha+\beta} f\right)(x)$;

(ii) $\left(D_{q}^{\alpha} I_{q}^{\alpha} f\right)(x)=f(x)$.

Lemma 2 (see [3], Theorem 2.4). Let $\alpha>0$ and $p$ be a positive integer. Then, the following equality holds:

$$
\left(I_{q}^{\alpha} D_{q}^{p} f\right)(x)=\left(D_{q}^{p} I_{q}^{\alpha} f\right)(x)-\sum_{k=0}^{p-1} \frac{x^{\alpha-p+k}}{\Gamma_{q}(\alpha+k-p+1)}\left(D_{q}^{k} f\right)(0) .
$$

Lemma 3. Suppose that (H1) holds, and $\alpha \in(2,3), v \in(1,2)$. If $g \in C[0,1]$, then the following boundary value problem

$$
\left\{\begin{array}{l}
D_{q}^{\alpha} x(t)+g(t)=0, t \in(0,1), \\
x(0)=0, D_{q} x(0)=0, D_{q}^{v} x(1)=\int_{0}^{1} h(t) D_{q}^{v} x(t) d_{q} t
\end{array}\right.
$$

has a unique solution

$$
x(t)=\int_{0}^{1} G(t, q s) g(s) d_{q} s,
$$

where

$$
\begin{aligned}
& G(t, q s)=G_{0}(t, q s)+\frac{t^{\alpha-1}}{A} \int_{0}^{1} h(t) G_{1}(t, q s) d_{q} t, \\
& G_{0}(t, q s)=\frac{1}{\Gamma_{q}(\alpha)} \begin{cases}t^{\alpha-1}(1-q s)^{(\alpha-v-1)}-(t-q s)^{(\alpha-1)}, & 0 \leq s \leq t \leq 1, \\
t^{\alpha-1}(1-q s)^{(\alpha-v-1)}, & 0 \leq t \leq s \leq 1,\end{cases} \\
& G_{1}(t, q s)=\frac{1}{\Gamma_{q}(\alpha)} \begin{cases}t^{\alpha-v-1}(1-q s)^{(\alpha-v-1)}-(t-q s)^{(\alpha-v-1)}, & 0 \leq s \leq t \leq 1, \\
t^{\alpha-v-1}(1-q s)^{(\alpha-v-1)}, & 0 \leq t \leq s \leq 1 .\end{cases}
\end{aligned}
$$

Proof. Using Definition 2 and Lemmas 2 and 3 we have

$$
x(t)=c_{1} t^{\alpha-1}+c_{2} t^{\alpha-2}+c_{3} t^{\alpha-3}-I_{q}^{\alpha} g(t), c_{i} \in \mathbb{R}, i=1,2,3 .
$$

Note from $x(0)=D_{q} x(0)=0$ we have $c_{2}=c_{3}=0$. Hence,

$$
x(t)=c_{1} t^{\alpha-1}-I_{q}^{\alpha} g(t),
$$

and

$$
D_{q}^{v} x(t)=\frac{\Gamma_{q}(\alpha)}{\Gamma_{q}(\alpha-v)} c_{1} t^{\alpha-v-1}-\frac{1}{\Gamma_{q}(\alpha-v)} \int_{0}^{t}(t-q s)^{(\alpha-v-1)} g(s) d_{q} s .
$$


Consequently, we have

$$
\begin{aligned}
D_{q}^{v} x(1) & =\frac{\Gamma_{q}(\alpha)}{\Gamma_{q}(\alpha-v)} c_{1}-\frac{1}{\Gamma_{q}(\alpha-v)} \int_{0}^{1}(1-q s)^{(\alpha-v-1)} g(s) d_{q} s \\
& =\int_{0}^{1} h(t) D_{q}^{v} x(t) d_{q} t \\
& =\frac{\Gamma_{q}(\alpha)}{\Gamma_{q}(\alpha-v)} c_{1} \int_{0}^{1} h(t) t^{\alpha-v-1} d_{q} t-\frac{1}{\Gamma_{q}(\alpha-v)} \int_{0}^{1} h(t) \int_{0}^{t}(t-q s)^{(\alpha-v-1)} g(s) d_{q} s d_{q} t .
\end{aligned}
$$

This, together with (H1), implies that

$$
c_{1}=\frac{1}{A \Gamma_{q}(\alpha)} \int_{0}^{1}(1-q s)^{(\alpha-v-1)} g(s) d_{q} s-\frac{1}{A \Gamma_{q}(\alpha)} \int_{0}^{1} h(t) \int_{0}^{t}(t-q s)^{(\alpha-v-1)} g(s) d_{q} s d_{q} t .
$$

Thus, we have

$$
\begin{aligned}
x(t)= & \frac{1}{A \Gamma_{q}(\alpha)} \int_{0}^{1} t^{\alpha-1}(1-q s)^{(\alpha-v-1)} g(s) d_{q} s-\frac{t^{\alpha-1}}{A \Gamma_{q}(\alpha)} \int_{0}^{1} h(t) \int_{0}^{t}(t-q s)^{(\alpha-v-1)} g(s) d_{q} s d_{q} t \\
& -\frac{1}{\Gamma_{q}(\alpha)} \int_{0}^{t}(t-q s)^{(\alpha-1)} g(s) d_{q} s \\
= & \frac{1}{A \Gamma_{q}(\alpha)} \int_{0}^{1} t^{\alpha-1}(1-q s)^{(\alpha-v-1)} g(s) d_{q} s-\frac{t^{\alpha-1}}{A \Gamma_{q}(\alpha)} \int_{0}^{1} h(t) \int_{0}^{t}(t-q s)^{(\alpha-v-1)} g(s) d_{q} s d_{q} t \\
& -\frac{1}{\Gamma_{q}(\alpha)} \int_{0}^{t}(t-q s)^{(\alpha-1)} g(s) d_{q} s+\frac{1}{\Gamma_{q}(\alpha)} \int_{0}^{1} t^{\alpha-1}(1-q s)^{(\alpha-v-1)} g(s) d_{q} s \\
& -\frac{1}{\Gamma_{q}(\alpha)} \int_{0}^{1} t^{\alpha-1}(1-q s)^{(\alpha-v-1)} g(s) d_{q} s \\
= & \int_{0}^{1} G_{0}(t, q s) g(s) d_{q} s+\frac{t^{\alpha-1}}{A \Gamma_{q}(\alpha)} \int_{0}^{1} h(t) t^{\alpha-v-1} d_{q} t \int_{0}^{1}(1-q s)^{(\alpha-v-1)} g(s) d_{q} s \\
& -\frac{t^{\alpha-1}}{A \Gamma_{q}(\alpha)} \int_{0}^{1} h(t) \int_{0}^{t}(t-q s)^{(\alpha-v-1)} g(s) d_{q} s d_{q} t \\
= & \int_{0}^{1} G_{0}(t, q s) g(s) d_{q} s+\frac{t^{\alpha-1}}{A \Gamma_{q}(\alpha)} \int_{0}^{1}\left[\int_{0}^{1} h(t) t^{\alpha-v-1}(1-q s)^{(\alpha-v-1)} d_{q} t-\int_{s}^{1} h(t)(t-q s)^{(\alpha-v-1)} d_{q} t\right] g(s) d_{q} s \\
= & \int_{0}^{1} G_{0}(t, q s) g(s) d_{q} s+\frac{t^{\alpha-1}}{A} \int_{0}^{1} \int_{0}^{1} h(t) G_{1}(t, q s) d_{q} t g(s) d_{q} s \\
= & \int_{0}^{1} G(t, q s) g(s) d_{q} s .
\end{aligned}
$$

This completes the proof.

Lemma 4 (see ([7], Lemma 2.2), ([4], Lemma 3.0.7), ([28], Lemma 2.7)). The functions $G_{i}(i=0,1)$ has the following properties

$$
\begin{aligned}
& \text { (i) } G_{i}(t, q s) \geq 0 \text { for } t, s \in[0,1] \\
& \text { (ii) } t^{\alpha-1} G_{0}(1, q s) \leq G_{0}(t, q s) \leq G_{0}(1, q s) \text { for } t, s \in[0,1] \text {. }
\end{aligned}
$$

Lemma 5. The function $G$ satisfies

$$
t^{\alpha-1} \varphi_{1}(q s) \leq G(t, q s) \leq \varphi_{1}(q s), \text { for } t, s \in[0,1]
$$

and

$$
G(t, q s) \leq t^{\alpha-1} \varphi_{2}(q s), \text { for } t, s \in[0,1]
$$


where

$$
\varphi_{1}(s)=G_{0}(1, s)+\frac{1}{A} \int_{0}^{1} h(t) G_{1}(t, s) d_{q} t, s \in[0,1]
$$

and

$$
\varphi_{2}(s)=\frac{1}{\Gamma_{q}(\alpha)}(1-s)^{(\alpha-v-1)}+\frac{1}{A} \int_{0}^{1} h(t) G_{1}(t, s) d_{q} t, s \in[0,1] .
$$

This is the direct result from Lemma 4 , so we omit the proof.

Let $E:=C[0,1],\|x\|:=\max _{t \in[0,1]}|x(t)|$ and $P:=\{x \in E: x(t) \geq 0, \forall t \in[0,1]\}$. Then $(E,\|\cdot\|)$ is a real Banach space and $P$ is a cone on $E$. Moreover, $E^{2}=E \times E$ is a Banach space with the norm $\|(u, v)\|=$ $\|u\|+\|v\|$, and $P^{2}=P \times P$ is a cone on $E^{2}$. From Lemma 3 we can define operators $T_{i}(i=1,2): E \rightarrow E$, and $T: E^{2} \rightarrow E^{2}$ as follows:

$$
\begin{aligned}
& \left(T_{1} y\right)(t):=\int_{0}^{1} G(t, q s) f_{1}(s, y(s)) d_{q} s, \\
& \left(T_{2} x\right)(t):=\int_{0}^{1} G(t, q s) f_{2}(s, x(s)) d_{q} s,
\end{aligned}
$$

and

$$
T(x, y)(t)=\left(\left(T_{1} y\right),\left(T_{2} x\right)\right)(t), t \in[0,1], x, y \in E,
$$

where $G$ is determined in Lemma 3. Please note that $T_{i}(i=1,2)$ and $T$ are completely continuous operators, and $(x, y)$ solves (8) if and only if $(x, y)$ is a fixed point of the operator $T$.

In addition, from Lemma 3 we can obtain that (9) is equivalent to

$$
x(t)=\lambda \int_{0}^{1} G(t, q s) x(s) d_{q} s, t \in[0,1] .
$$

For our purposes, we need to define the operator $L$ by

$$
(L x)(t)=\int_{0}^{1} G(t, q s) x(s) d_{q} s, t \in[0,1], x \in E .
$$

It is not difficult to prove that $L: E \rightarrow E$ is a linear completely continuous and $T(P) \subset P$. From Lemmas 2 and 3 in [7] we obtain that the spectral radius, denoted by $r(L)$, is not equal to 0 , and $L$ has a positive eigenfunction $\varphi^{*}$ corresponding to its first eigenvalue $\lambda_{1}=(r(L))^{-1}$, i.e., $\varphi^{*}=\lambda_{1} L \varphi^{*}$.

Lemma 6. Let $P_{0}=\left\{x \in P: x(t) \geq t^{\alpha-1}\|x\|, \forall t \in[0,1]\right\}$. Then $L(P) \subset P_{0}$.

Proof. If $x \in P$, and from (11) we have

$$
t^{\alpha-1} \int_{0}^{1} \varphi_{1}(q s) x(s) d_{q} s \leq \int_{0}^{1} G(t, q s) x(s) d_{q} s \leq \int_{0}^{1} \varphi_{1}(q s) x(s) d_{q} s, \text { for } t \in[0,1] .
$$

Therefore, we have

$$
(L x)(t) \geq t^{\alpha-1} \int_{0}^{1} \varphi_{1}(q s) x(s) d_{q} s \geq t^{\alpha-1}\|L x\|, \text { for } t \in[0,1] .
$$

This completes the proof.

Remark 2. From Lemma 6 we have $\varphi^{*} \in P_{0}$.

We recall the following topological degree theorems, which will play important roles in proving our main results. 
Lemma 7 (see ([36], Theorem A.3.3)). [ Let $\Omega$ be a bounded open set in a Banach space E, and $T: \Omega \rightarrow E a$ continuous compact operator. If there exists $x_{0} \in E \backslash\{0\}$ such that

$$
x-T x \neq \mu x_{0}, \forall x \in \partial \Omega, \mu \geq 0,
$$

then the topological degree $\operatorname{deg}(I-T, \Omega, 0)=0$.

Lemma 8 (see ([36], Lemma 2.5.1)). Let $\Omega$ be a bounded open set in a Banach space $E$ with $0 \in \Omega$, and $T: \Omega \rightarrow E$ a continuous compact operator. If

$$
T x \neq \mu x, \forall x \in \partial \Omega, \mu \geq 1,
$$

then the topological degree $\operatorname{deg}(I-T, \Omega, 0)=1$.

\section{Proof of Theorem 1}

In this section, we present the detailed proof of Theorem 1 . From (H6) there exist $\varepsilon_{0} \in\left(0, \lambda_{1}\right)$ and $r_{1}>0$ such that

$$
\left|f_{1}(t, y)\right| \leq\left(\lambda_{1}-\varepsilon_{0}\right)|y|,\left|f_{2}(t, x)\right| \leq\left(\lambda_{1}-\varepsilon_{0}\right)|x|, \forall t \in[0,1], x, y \in \mathbb{R} \text { with }|x|,|y| \leq r_{1}
$$

This implies that

$$
\left|\left(T_{1} y\right)(t)\right| \leq \int_{0}^{1} G(t, q s)\left|f_{1}(s, y(s))\right| d_{q} s \leq\left(\lambda_{1}-\varepsilon_{0}\right) \int_{0}^{1} G(t, q s)|y(s)| d_{q} s,
$$

and

$$
\left|\left(T_{2} x\right)(t)\right| \leq \int_{0}^{1} G(t, q s)\left|f_{2}(s, x(s))\right| d_{q} s \leq\left(\lambda_{1}-\varepsilon_{0}\right) \int_{0}^{1} G(t, q s)|x(s)| d_{q} s .
$$

Now we prove that

$$
(x, y) \neq \mu T(x, y) \text { for all } x, y \in \partial B_{r_{1}} \text { and } \mu \in[0,1]
$$

We argue by contradiction. Suppose there exist $x, y \in \partial B_{r_{1}}$ and $\mu \in[0,1]$ such that

$$
(x, y)=\mu T(x, y)
$$

Therefore,

$$
x=\mu T_{1} y, \text { and } y=\mu T_{2} x .
$$

This implies that

$$
|x(t)|=\mu\left|\left(T_{1} y\right)(t)\right| \leq\left(\lambda_{1}-\varepsilon_{0}\right) \int_{0}^{1} G(t, q s)|y(s)| d_{q} s, t \in[0,1],
$$

and

$$
|y(t)|=\mu\left|\left(T_{2} x\right)(t)\right| \leq\left(\lambda_{1}-\varepsilon_{0}\right) \int_{0}^{1} G(t, q s)|x(s)| d_{q} s, t \in[0,1] .
$$

Consequently, we have

$$
|x(t)|+|y(t)| \leq\left(\lambda_{1}-\varepsilon_{0}\right) \int_{0}^{1} G(t, q s)(|x(s)|+|y(s)|) d_{q} s, t \in[0,1] .
$$

Let $z(t)=|x(t)|+|y(t)|$. 
Then $z \in P$ and

$$
z(t) \leq\left(\lambda_{1}-\varepsilon_{0}\right) \int_{0}^{1} G(t, q s) z(s) d_{q} s=\left(\lambda_{1}-\varepsilon_{0}\right)(L z)(t), t \in[0,1] .
$$

The $n$th iteration of this inequality shows that $z(t) \leq\left(\lambda_{1}-\varepsilon_{0}\right)^{n}\left(L^{n} z\right)(t)(n=1,2, \ldots)$, and then $\|z\| \leq\left(\lambda_{1}-\varepsilon_{0}\right)^{n}\left\|L^{n}\right\| \cdot\|z\|$, i.e., $1 \leq\left(\lambda_{1}-\varepsilon_{0}\right)^{n}\left\|L^{n}\right\|$.

This yields

$$
1 \leq\left(\lambda_{1}-\varepsilon_{0}\right) \lim _{n \rightarrow \infty} \sqrt[n]{\left\|L^{n}\right\|}=\left(\lambda_{1}-\varepsilon_{0}\right) r(L)=\frac{\lambda_{1}-\varepsilon_{0}}{\lambda_{1}}<1,
$$

which is a contradiction. Hence, (14) holds. It follows from Lemma 8 that

$$
\operatorname{deg}\left(I-T, B_{r_{1}}, 0\right)=1 .
$$

On the other hand, from (H5) there exist $\varepsilon_{1}>0$ and $r_{2}>0$ such that

$$
f_{1}(t, y) \geq\left(\lambda_{1}+\varepsilon_{1}\right)|y|, f_{2}(t, x) \geq\left(\lambda_{1}+\varepsilon_{1}\right)|x|, \forall t \in[0,1],|x|,|y|>r_{2} .
$$

Let $M_{1}=\max _{t \in[0,1],|y| \leq r_{2}}\left[\left|f_{1}(t, y)\right|+\left(\lambda_{1}+\varepsilon_{1}\right)|y|\right], M_{2}=\max _{t \in[0,1],|x| \leq r_{2}}\left[\left|f_{2}(t, x)\right|+\left(\lambda_{1}+\varepsilon_{1}\right)|x|\right]$. Then

$$
f_{1}(t, y) \geq\left(\lambda_{1}+\varepsilon_{1}\right)|y|-M_{1}, f_{2}(t, x) \geq\left(\lambda_{1}+\varepsilon_{1}\right)|x|-M_{2}, \forall t \in[0,1], x, y \in R .
$$

For any given $\varepsilon, \widetilde{\varepsilon}$ with $\varepsilon_{1}-\left\|c_{1}\right\| \varepsilon>0, \varepsilon_{1}-\left\|c_{2}\right\| \widetilde{\varepsilon}>0$, and from (H4) there exists $r_{3}>r_{2}$ such that

$$
K_{1}(y) \leq \varepsilon|y|, K_{2}(x) \leq \widetilde{\varepsilon}|x|, \forall|x|,|y|>r_{3} .
$$

Let $K_{1}^{*}=\max _{|y| \leq r_{3}} K_{1}(y)$, and $K_{2}^{*}=\max _{|x| \leq r_{3}} K_{2}(x)$. Then we have

$$
K_{1}(y) \leq \varepsilon|y|+K_{1}^{*}, K_{2}(x) \leq \widetilde{\varepsilon}|x|+K_{2}^{*}, \forall x, y \in \mathbb{R} .
$$

Please note that $\varepsilon, \widetilde{\varepsilon}$ can be chosen arbitrarily small, so we can let $R_{1}>\max \left\{r_{1}, N_{1}, N_{2}, N_{3}, N_{4}\right\}$, where $r_{1}$ is defined by (14), and

$$
\begin{aligned}
& N_{1}=\frac{\left(2\left\|b_{1}\right\|+2\left\|c_{1}\right\| K_{1}^{*}+M_{1}\right) \int_{0}^{1} \varphi_{1}(q s) d_{q} s}{\frac{1}{2}-\varepsilon\left\|c_{1}\right\| \int_{0}^{1} \varphi_{1}(q s) d_{q} s}, \\
& N_{2}=\frac{\left(2\left\|b_{2}\right\|+2\left\|c_{2}\right\| K_{2}^{*}+M_{2}\right) \int_{0}^{1} \varphi_{1}(q s) d_{q} s}{\frac{1}{2}-\widetilde{\varepsilon}\left\|c_{2}\right\| \int_{0}^{1} \varphi_{1}(q s) d_{q} s}, \\
& N_{3}=\frac{N_{5}\left(2\left\|b_{1}\right\|+2\left\|b_{2}\right\|+2\left\|c_{1}\right\| K_{1}^{*}+2\left\|c_{2}\right\| K_{2}^{*}+M_{1}+M_{2}\right)}{\left(\varepsilon_{1}-\left\|c_{1}\right\| \varepsilon\right)-N_{5}\left(\left\|c_{1}\right\| \varepsilon+\left\|c_{2}\right\| \widetilde{\varepsilon}\right)}, \\
& N_{4}=\frac{N_{6}\left(2\left\|b_{1}\right\|+2\left\|b_{2}\right\|+2\left\|c_{1}\right\| K_{1}^{*}+2\left\|c_{2}\right\| K_{2}^{*}+M_{1}+M_{2}\right)}{\left(\varepsilon_{1}-\left\|c_{2}\right\| \widetilde{\varepsilon}\right)-N_{6}\left(\left\|c_{1}\right\| \varepsilon+\left\|c_{2}\right\| \widetilde{\varepsilon}\right)}, \\
& N_{5}=\left(\varepsilon_{1}-\left\|c_{1}\right\| \varepsilon\right) \int_{0}^{1} \varphi_{1}(q s) d_{q} s+\left(\lambda_{1}+\varepsilon_{1}-\left\|c_{1}\right\| \varepsilon\right) \int_{0}^{1} \varphi_{2}(q s) d_{q} s, \\
& N_{6}=\left(\varepsilon_{1}-\left\|c_{2}\right\| \widetilde{\varepsilon}\right) \int_{0}^{1} \varphi_{1}(q s) d_{q} s+\left(\lambda_{1}+\varepsilon_{1}-\left\|c_{2}\right\| \widetilde{\varepsilon}\right) \int_{0}^{1} \varphi_{2}(q s) d_{q} s .
\end{aligned}
$$

Now we prove that

$$
(x, y)-T(x, y) \neq \mu\left(\varphi^{*}, \varphi^{*}\right), \forall x, y \in \partial B_{R_{1}}, \mu \geq 0,
$$


where $\varphi^{*}$ is the positive eigenfunction of $L$ corresponding to the eigenvalue $\lambda_{1}$. We argue by contradiction. Suppose there exist $x, y \in \partial B_{R_{1}}$ and $\mu \geq 0$ such that

$$
(x, y)-T(x, y)=\mu\left(\varphi^{*}, \varphi^{*}\right),
$$

and thus

$$
x=T_{1} y+\mu \varphi^{*}, y=T_{2} x+\mu \varphi^{*} .
$$

Let

$$
\widetilde{x}(t)=\int_{0}^{1} G(t, q s)\left[2 b_{2}(s)+c_{2}(s) K_{2}(x(s))+M_{2}+\left\|c_{2}\right\| K_{2}^{*}\right] d_{q} s,
$$

and

$$
\widetilde{y}(t)=\int_{0}^{1} G(t, q s)\left[2 b_{1}(s)+c_{1}(s) K_{1}(y(s))+M_{1}+\left\|c_{1}\right\| K_{1}^{*}\right] d_{q} s .
$$

Now we estimate the norms $\|\widetilde{x}\|$ and $\|\widetilde{y}\|$. Please note that $\|x\|=\|y\|=R_{1}$, and from (17) we have

$$
\begin{aligned}
\|\widetilde{y}\| & \leq \int_{0}^{1} \varphi_{1}(q s)\left[2\left\|b_{1}\right\|+\left\|c_{1}\right\|\left(\varepsilon\|y\|+K_{1}^{*}\right)+M_{1}+\left\|c_{1}\right\| K_{1}^{*}\right] d_{q} s \\
& =\int_{0}^{1} \varphi_{1}(q s) d_{q} s \times\left(2\left\|b_{1}\right\|+2\left\|c_{1}\right\| K_{1}^{*}+M_{1}+\left\|c_{1}\right\| \varepsilon R_{1}\right) \\
& <\frac{1}{2} R_{1}
\end{aligned}
$$

and

$$
\begin{aligned}
\|\widetilde{x}\| & \leq \int_{0}^{1} \varphi_{1}(q s)\left[2\left\|b_{2}\right\|+\left\|c_{2}\right\|\left(\widetilde{\varepsilon}\|x\|+K_{2}^{*}\right)+M_{2}+\left\|c_{2}\right\| K_{2}^{*}\right] d_{q} s \\
& =\int_{0}^{1} \varphi_{1}(q s) d_{q} s \times\left(2\left\|b_{2}\right\|+2\left\|c_{2}\right\| K_{2}^{*}+M_{2}+\left\|c_{2}\right\| \widetilde{\varepsilon} R_{1}\right) \\
& <\frac{1}{2} R_{1} .
\end{aligned}
$$

Furthermore, from (H3) and Lemma 6 we have $\widetilde{x}, \widetilde{y} \in P_{0}$. Consequently, we obtain

$$
\begin{aligned}
x(t)+\widetilde{y}(t) & =\left(T_{1} y\right)(t)+\mu \varphi^{*}(t)+\widetilde{y}(t) \\
& =\int_{0}^{1} G(t, q s)\left[f_{1}(s, y(s))+2 b_{1}(s)+c_{1}(s) K_{1}(y(s))+M_{1}+\left\|c_{1}\right\| K_{1}^{*}\right] d_{q} s+\mu \varphi^{*}(t),
\end{aligned}
$$

and

$$
\begin{aligned}
y(t)+\widetilde{x}(t) & =\left(T_{2} x\right)(t)+\mu \varphi^{*}(t)+\widetilde{x}(t) \\
& =\int_{0}^{1} G(t, q s)\left[f_{2}(s, x(s))+2 b_{2}(s)+c_{2}(s) K_{2}(x(s))+M_{2}+\left\|c_{2}\right\| K_{2}^{*}\right] d_{q} s+\mu \varphi^{*}(t) .
\end{aligned}
$$

Using (H3), Lemma 6 and Remark 2 we have

$$
x+\widetilde{y} \in P_{0}, y+\widetilde{x} \in P_{0} .
$$

Please note that $\|x\|=\|y\|=R_{1}, y+\widetilde{y}+\widetilde{x} \in P_{0}$, and $x+\widetilde{y}+\widetilde{x} \in P_{0}$. Therefore, we get

$$
x(t)+\widetilde{y}(t)+\widetilde{x}(t) \geq t^{\alpha-1}\|x+\widetilde{y}+\widetilde{x}\| \geq t^{\alpha-1}(\|x\|-\|\widetilde{y}+\widetilde{x}\|) \geq t^{\alpha-1}[\|x\|-(\|\widetilde{y}\|+\|\widetilde{x}\|)],
$$

and

$$
y(t)+\widetilde{y}(t)+\widetilde{x}(t) \geq t^{\alpha-1}\|y+\widetilde{y}+\widetilde{x}\| \geq t^{\alpha-1}(\|y\|-\|\widetilde{y}+\widetilde{x}\|) \geq t^{\alpha-1}[\|y\|-(\|\widetilde{y}\|+\|\widetilde{x}\|)] .
$$


Consequently, note that the range value of $R_{1}$, from (22) and (23) we find

$$
\begin{aligned}
& \left(\varepsilon_{1}-\left\|c_{1}\right\| \varepsilon\right) \int_{0}^{1} G(t, q s)(y(s)+\widetilde{y}(s)+\widetilde{x}(s)) d_{q} s-\left(\lambda_{1}+\varepsilon_{1}-\left\|c_{1}\right\| \varepsilon\right) \int_{0}^{1} G(t, q s)(\widetilde{y}(s)+\widetilde{x}(s)) d_{q} s \\
& \geq\left(\varepsilon_{1}-\left\|c_{1}\right\| \varepsilon\right) \int_{0}^{1} G(t, q s) s^{\alpha-1}\left(R_{1}-(\|\widetilde{y}\|+\|\widetilde{x}\|)\right) d_{q} s-\left(\lambda_{1}+\varepsilon_{1}-\left\|c_{1}\right\| \varepsilon\right) \int_{0}^{1} G(t, q s) \\
& \quad \int_{0}^{1} G(s, q \tau)\left[2 b_{1}(\tau)+2 b_{2}(\tau)+c_{1}(\tau) K_{1}(y(\tau))+c_{2}(\tau) K_{2}(x(\tau))+M_{1}+M_{2}+\left\|c_{1}\right\| K_{1}^{*}+\left\|c_{2}\right\| K_{2}^{*}\right] d_{q} \tau d_{q} s \\
& \geq\left(\varepsilon_{1}-\left\|c_{1}\right\| \varepsilon\right) \int_{0}^{1} G(t, q s) s^{\alpha-1}\left(R_{1}-(\|\widetilde{y}\|+\|\widetilde{x}\|)\right) d_{q} s-\left(\lambda_{1}+\varepsilon_{1}-\left\|c_{1}\right\| \varepsilon\right) \int_{0}^{1} G(t, q s) \\
& \quad \int_{0}^{1} s^{\alpha-1} \varphi_{2}(q \tau)\left[2\left\|b_{1}\right\|+2\left\|b_{2}\right\|+\left\|c_{1}\right\|\left(\varepsilon\|y\|+K_{1}^{*}\right)+\left\|c_{2}\right\|\left(\widetilde{\varepsilon}\|x\|+K_{2}^{*}\right)+M_{1}+M_{2}+\left\|c_{1}\right\| K_{1}^{*}+\left\|c_{2}\right\| K_{2}^{*}\right] d_{q} \tau d_{q} s \\
& \geq 0
\end{aligned}
$$

and

$$
\begin{aligned}
& \left(\varepsilon_{1}-\left\|c_{2}\right\| \widetilde{\mathcal{\varepsilon}}\right) \int_{0}^{1} G(t, q s)(x(s)+\widetilde{x}(s)+\widetilde{y}(s)) d_{q} s-\left(\lambda_{1}+\varepsilon_{1}-\left\|c_{2}\right\| \widetilde{\varepsilon}\right) \int_{0}^{1} G(t, q s)(\widetilde{x}(s)+\widetilde{y}(s)) d_{q} s \\
& \geq\left(\varepsilon_{1}-\left\|c_{2}\right\| \widetilde{\mathcal{E}}\right) \int_{0}^{1} G(t, q s) s^{\alpha-1}\left(R_{1}-(\|\widetilde{y}\|+\|\widetilde{x}\|)\right) d_{q} s-\left(\lambda_{1}+\varepsilon_{1}-\left\|c_{2}\right\| \widetilde{\mathcal{E}}\right) \int_{0}^{1} G(t, q s) \\
& \quad \int_{0}^{1} s^{\alpha-1} \varphi_{2}(q \tau)\left[2\left\|b_{1}\right\|+2\left\|b_{2}\right\|+\left\|c_{1}\right\|\left(\varepsilon\|y\|+K_{1}^{*}\right)+\left\|c_{2}\right\|\left(\widetilde{\varepsilon}\|x\|+K_{2}^{*}\right)+M_{1}+M_{2}+\left\|c_{1}\right\| K_{1}^{*}+\left\|c_{2}\right\| K_{2}^{*}\right] d_{q} \tau d_{q} s \\
& \geq 0 .
\end{aligned}
$$

Consequently, from (16) and (17) we have

$$
\begin{aligned}
& \int_{0}^{1} G(t, q s)\left[f_{1}(s, y(s))+2 b_{1}(s)+c_{1}(s) K_{1}(y(s))+M_{1}+\left\|c_{1}\right\| K_{1}^{*}\right] d_{q} s \\
& \geq \int_{0}^{1} G(t, q s)\left[\left(\lambda_{1}+\varepsilon_{1}\right)|y(s)|-M_{1}-b_{1}(s)-c_{1}(s)\left(\varepsilon|y(s)|+K_{1}^{*}\right)+b_{1}(s)+M_{1}+\left\|c_{1}\right\| K_{1}^{*}\right] d_{q} s \\
& \geq \int_{0}^{1} G(t, q s)\left[\left(\lambda_{1}+\varepsilon_{1}\right)|y(s)|-\left\|c_{1}\right\|\left(\varepsilon|y(s)|+K_{1}^{*}\right)+\left\|c_{1}\right\| K_{1}^{*}\right] d_{q} s \\
& =\left(\lambda_{1}+\varepsilon_{1}-\left\|c_{1}\right\| \varepsilon\right) \int_{0}^{1} G(t, q s)|y(s)| d_{q} s \\
& \geq\left(\lambda_{1}+\varepsilon_{1}-\left\|c_{1}\right\| \varepsilon\right) \int_{0}^{1} G(t, q s) y(s) d_{q} s \\
& =\left(\lambda_{1}+\varepsilon_{1}-\left\|c_{1}\right\| \varepsilon\right) \int_{0}^{1} G(t, q s)(y(s)+\widetilde{y}(s)+\widetilde{x}(s)) d_{q} s-\left(\lambda_{1}+\varepsilon_{1}-\left\|c_{1}\right\| \varepsilon\right) \int_{0}^{1} G(t, q s)(\widetilde{y}(s)+\widetilde{x}(s)) d_{q} s \\
& \geq \lambda_{1} L(y+\widetilde{y}+\widetilde{x})(t) \\
& \geq \lambda_{1} L(y+\widetilde{x})(t),
\end{aligned}
$$

and

$$
\begin{aligned}
& \int_{0}^{1} G(t, q s)\left[f_{2}(s, x(s))+2 b_{2}(s)+c_{2}(s) K_{2}(x(s))+M_{2}+\left\|c_{2}\right\| K_{2}^{*}\right] d_{q} s \\
& \geq \int_{0}^{1} G(t, q s)\left[\left(\lambda_{1}+\varepsilon_{1}\right)|x(s)|-M_{2}-b_{2}(s)-c_{2}(s)\left(\widetilde{\varepsilon}|x(s)|+K_{2}^{*}\right)+b_{2}(s)+M_{2}+\left\|c_{2}\right\| K_{2}^{*}\right] d_{q} s \\
& \geq \int_{0}^{1} G(t, q s)\left[\left(\lambda_{1}+\varepsilon_{1}\right)|x(s)|-\left\|c_{2}\right\|\left(\widetilde{\varepsilon}|x(s)|+K_{2}^{*}\right)+\left\|c_{2}\right\| K_{2}^{*}\right] d_{q} s \\
& \geq\left(\lambda_{1}+\varepsilon_{1}-\left\|c_{2}\right\| \widetilde{\varepsilon}\right) \int_{0}^{1} G(t, q s) x(s) d_{q} s \\
& =\left(\lambda_{1}+\varepsilon_{1}-\left\|c_{2}\right\| \widetilde{\varepsilon}\right) \int_{0}^{1} G(t, q s)(x(s)+\widetilde{x}(s)+\widetilde{y}(s)) d_{q} s-\left(\lambda_{1}+\varepsilon_{1}-\left\|c_{2}\right\| \widetilde{\varepsilon}\right) \int_{0}^{1} G(t, q s)(\widetilde{x}(s)+\widetilde{y}(s)) d_{q} s \\
& \geq \lambda_{1} L(x+\widetilde{x}+\widetilde{y})(t) \\
& \geq \lambda_{1} L(x+\widetilde{y})(t) .
\end{aligned}
$$


Now, using (26)-(27) we have

$$
T_{1} y+\widetilde{y} \geq \lambda_{1} L(y+\widetilde{x}), T_{2} x+\widetilde{x} \geq \lambda_{1} L(x+\widetilde{y}) .
$$

Thus, from (19) we have

$$
x+y+\widetilde{x}+\widetilde{y}=T_{1} y+T_{2} x+\widetilde{x}+\widetilde{y}+2 \mu \varphi^{*} \geq \lambda_{1} L(x+y+\widetilde{x}+\widetilde{y})+2 \mu \varphi^{*} \geq 2 \mu \varphi^{*} .
$$

Define $\mu^{*}=\sup S_{\mu}:=\sup \left\{\mu>0: x+y+\widetilde{x}+\widetilde{y} \geq 2 \mu \varphi^{*}\right\}$. Then $S_{\mu}(\neq \varnothing)$ is a limited set, $\mu^{*} \geq \mu$ and $x+y+\widetilde{x}+\widetilde{y} \geq 2 \mu^{*} \varphi^{*}$. From $\varphi^{*}=\lambda_{1} L \varphi^{*}$, we obtain

$$
\lambda_{1} L(x+y+\widetilde{x}+\widetilde{y}) \geq \lambda_{1} L\left(2 \mu^{*} \varphi^{*}\right)=2 \mu^{*} \lambda_{1} L \varphi^{*}=2 \mu^{*} \varphi^{*} .
$$

Hence

$$
x+y+\widetilde{x}+\widetilde{y} \geq \lambda_{1} L(x+y+\widetilde{x}+\widetilde{y})+2 \mu \varphi^{*} \geq 2\left(\mu+\mu^{*}\right) \varphi^{*},
$$

which contradicts the definition of $\mu^{*}$. Therefore, (18) holds, and from Lemma 7 we obtain

$$
\operatorname{deg}\left(I-T, B_{R_{1}}, 0\right)=0 .
$$

Now (15) and (28) together imply that

$$
\operatorname{deg}\left(I-T, B_{R_{1}} \backslash \bar{B}_{r_{1}}, 0\right)=\operatorname{deg}\left(I-T, B_{R_{1}}, 0\right)-\operatorname{deg}\left(I-T, B_{r_{1}}, 0\right)=-1 .
$$

Therefore the operator $T$ has at least one fixed point in $B_{R_{1}} \backslash \bar{B}_{r_{1}}$. Equivalently, (8) has at least one nontrivial solution. This completes the proof.

\section{Conclusions}

In this paper, we use topological degree to study nontrivial solutions for the system of fractional $q$-difference Equation (8) with $q$-integral boundary conditions. There are only a few papers in the literature which consider systems of fractional $q$-difference equations with $q$-integral boundary conditions where the nonlinear terms may be unbounded from below. Our main theorem is obtained under some conditions concerning the first eigenvalues corresponding to the relevant linear operators. As a result, our main result generalizes and improves the corresponding ones in the works cited in this paper.

Author Contributions: Conceptualization, Y.L., J.L., J.X. and D.O.; methodology, Y.L., J.L., J.X. and D.O.; software, Y.L., J.L.; validation, Y.L., J.L., J.X. and D.O.; formal analysis, Y.L., J.L., J.X. and D.O.; investigation, J.L., J.X. and D.O.; resources, Y.L., J.X.; data curation, Y.L., J.X.; writing original draft preparation, J.L., J.X. and D.O.; writing review and editing, Y.L., J.L., J.X. and D.O.; visualization, Y.L., J.X. and D.O.; supervision, J.X. and D.O.; project administration, J.X.; funding acquisition, Y.L., J.X. All authors have read and agreed to the published version of the manuscript.

Funding: This work is supported by the China Postdoctoral Science Foundation (Grant No. 2019M652348), Technology Research Foundation of Chongqing Educational Committee(Grant No. KJQN201900539), Key Research Funds for the Universities of Henan Province (19A110018, 20B110006), Fundamental Research Funds for the Universities of Henan Province (NSFRF180320) and Henan Polytechnic University Doctor Fund (No. B2016-58), Outstanding Young Foundation of Anhui Provincial Education Department (Grant No. gxyqZD2016339), Natural Science Foundation of Anhui Provincial Education Department (Grant No. KJ2018A0452).

Conflicts of Interest: The authors declare no conflict of interest.

\section{References}

1. Jackson, F. On $q$-functions and a certain difference operator. Trans. R. Soc. Edinb. 1908, 46, 253-281. [CrossRef]

2. Jackson, F. On q-definite integrals. Quart J. Pure Appl. Math. 1910, 41, 193-203. 
3. Ferreira, R.A.C. Nontrivial solutions for fractional $q$-difference boundary value problems. Electron. J. Qual. Theory Differ. Equ. 2010, 70, 1-10. [CrossRef]

4. Ferreira, R.A.C. Positive solutions for a class of boundary value problems with fractional $q$-differences. Comput. Math. Appl. 2011, 61,367-373. [CrossRef]

5. Yang, C. Positive solutions for a three-point boundary value problem of fractional $q$-difference equations. Symmetry 2018, 10, 358. [CrossRef]

6. Zhao, Y.; Chen, H.; Zhang, Q. Existence and multiplicity of positive solutions for nonhomogeneous boundary value problems with fractional $q$-derivatives. Bound. Value Probl. 2013, 2013, 103. [CrossRef]

7. Ma, K.; Han, Z.; Sun, S. Existence and uniqueness of solutions for fractional $q$-difference Schrödinger equations. J. Appl. Math. Comput. 2020, 62, 611-620. [CrossRef]

8. Guo, F.; Kang, S. Positive solutions for a class of fractional boundary value problem with $q$-derivatives. Mediterr. J. Math. 2019, 16, 113. [CrossRef]

9. Jin, N.; Sun, S.; Chen, G. Existence of solutions for a class of the boundary value problem of fractional q-difference inclusions. J. Appl. Math. Comput. 2017, 55, 409-420. [CrossRef]

10. Kang, S.; Chen, H.; Li, L.; Cui, Y.; Ma, S. Existence of three positive solutions for a class of Riemann-Liouville fractional $q$-difference equation. J. Anal. Appl. Comput. 2019, 9, 590-600.

11. Zhai, C.; Ren, J. The unique solution for a fractional $q$-difference equation with three-point boundary conditions. Indag. Math. 2018, 29, 948-961. [CrossRef]

12. Zhai, C.; Ren, J. Nonlocal $q$-fractional boundary value problem with Stieltjes integral conditions. Nonlinear Anal. Model. Control 2019, 24, 582-602.

13. Ren, J.; Zhai, C. Unique solutions for fractional $q$-difference boundary value problems via a fixed point method. Bull. Malays. Math. Sci. Soc. 2019, 42, 1507-1521. [CrossRef]

14. Almeida, R.; Martins, N. Existence results for fractional $q$-difference equations of order alpha is an element of ]2,3 [ with three-point boundary conditions. Commun. Nonlinear Sci. Numer. Simul. 2014, 19, 1675-1685. [CrossRef]

15. Ahmad, B.; Nieto, J.J.; Alsaedi, A.; Al-Hutami, H. Existence of solutions for nonlinear fractional q-difference integral equations with two fractional orders and nonlocal four-point boundary conditions. J. Franklin Inst. 2014, 351, 2890-2909. [CrossRef]

16. Ahmad, B.; Nieto, J.J.; Alsaedi, A.; Al-Hutami, H. Boundary value problems of nonlinear fractional $q$-difference (integral) equations with two fractional orders and four-point nonlocal integral boundary conditions. Filomat 2014, 28, 1719-1736. [CrossRef]

17. Mao, J.; Zhao, Z.; Wang, C. The unique iterative positive solution of fractional boundary value problem with q-difference. Appl. Math. Lett. 2020, 100, 106002. [CrossRef]

18. Yang, W. Existence results for nonlinear fractional $q$-difference equations with nonlocal Riemann-Liouville $q$-integral boundary conditions. Filomat 2016, 30, 2521-2533. [CrossRef]

19. Yang, W.; Qin, Y. Positive solutions for nonlinear Caputo type fractional $q$-difference equations with integral boundary conditions. Mathematics 2016, 4, 63. [CrossRef]

20. Wang, G. Twin iterative positive solutions of fractional $q$-difference Schrödinger equations. Appl. Math. Lett. 2018, 76, 103-109. [CrossRef]

21. Wang, G.; Bai, Z.; Zhang, L. Successive iterations for unique positive solution of a nonlinear fractional $q$-integral boundary value problem. J. Anal. Appl. Comput. 2019, 9, 1204-1215. [CrossRef]

22. Nasiruzzaman, M.; Mukheimer, A.; Mursaleen, M. A Dunkl-type generalization of Szász-Kantorovich operators via Post-Quantum calculus. Symmetry 2019, 11, 232. [CrossRef]

23. Bai, C.; Yang, D. The iterative positive solution for a system of fractional $q$-difference equations with four-point boundary conditions. Discret. Dyn. Nat. Soc. 2020, 2020, 3970903. [CrossRef]

24. Suantai, S.; Ntouyas, S.K.; Asawasamrit, S.; Tariboon, J. A coupled system of fractional $q$-integro-difference equations with nonlocal fractional $q$-integral boundary conditions. Adv. Differ. Equ. 2015, 2015, 124. [CrossRef]

25. Jiang, M.; Zhong, S. Existence of extremal solutions for a nonlinear fractional $q$-difference system. Mediterr. J. Math. 2016, 13, 279-299. [CrossRef]

26. Yang, W. Positive solutions for nonlinear semipositone fractional $q$-difference system with coupled integral boundary conditions. Appl. Math. Comput. 2014, 244, 702-725. [CrossRef] 
27. Zhao, Q.; Yang, W. Positive solutions for singular coupled integral boundary value problems of nonlinear higher-order fractional $q$-difference equations. Adv. Differ. Equ. 2015, 2015, 290. [CrossRef]

28. Cheng, W.; Xu, J.; Cui, Y. Positive solutions for a system of nonlinear semipositone fractional $q$-difference equations with $q$-integral boundary conditions. J. Nonlinear Sci. Appl. 2017, 10, 4430-4440. [CrossRef]

29. Fu, Z.; Bai, S.; O’Regan, D.; Xu, J. Nontrivial solutions for an integral boundary value problem involving Riemann-Liouville fractional derivatives. J. Inequal. Appl. 2019, 2019, 104. [CrossRef]

30. Xu, J.; Goodrich, C.S.; Cui, Y. Positive solutions for a system of first-order discrete fractional boundary value problems with semipositone nonlinearities. Rev. R. Acad. Cienc. Exactas Fis. Nat. Ser. A Mater. 2019, 113, 1343-1358. [CrossRef]

31. Zhang, H.; Li, Y.; Xu, J. Positive solutions for a system of fractional integral boundary value problems involving Hadamard-type fractional derivatives. Complexity 2019, 2019, 2671539. [CrossRef]

32. Xu, J.; Wei, Z.; O’Regan, D.; Cui, Y. Infinitely many solutions for fractional Schrödinger-Maxwell equations. J. Anal. Appl. Comput. 2019, 9, 1165-1182. [CrossRef]

33. Cheng, W.; Xu, J.; O’Regan, D.; Cui, Y. Positive solutions for a nonlinear discrete fractional boundary value problems with a $p$-Laplacian operator. J. Anal. Appl. Comput. 2019, 9, 1959-1972.

34. Cheng, W.; Xu, J.; Cui, Y.; Ge, Q. Positive solutions for a class of fractional difference systems with coupled boundary conditions. Adv. Differ. Equ. 2019, 2019, 249. [CrossRef]

35. Xu, J.; Jiang, J.; O'Regan, D. Positive solutions for a class of $p$-Laplacian Hadamard fractional-order three-point boundary value problems. Mathematics 2020, 8, 308. [CrossRef]

36. Guo, D.; Lakshmikantham, V. Nonlinear Problems in Abstract Cones; Academic Press: Orlando, FL, USA, 1988.

(C) 2020 by the authors. Licensee MDPI, Basel, Switzerland. This article is an open access article distributed under the terms and conditions of the Creative Commons Attribution (CC BY) license (http:// creativecommons.org/licenses/by/4.0/). 